\title{
Reliability Model of Mechanical Components with Dependent Failure Modes
}

\author{
Shijie Wang \\ School of Mechanical Engineering, Shenyang University of Technology, Shenyang, Liaoning 110870, China \\ Correspondence should be addressed to Shijie Wang; jzlsbw@163.com
}

Received 27 July 2012; Revised 15 December 2012; Accepted 17 December 2012

Academic Editor: M. Onder Efe

Copyright (C) 2013 Shijie Wang. This is an open access article distributed under the Creative Commons Attribution License, which permits unrestricted use, distribution, and reproduction in any medium, provided the original work is properly cited.

\begin{abstract}
A mechanical component may fail in many modes that are usually not independent. There is generally not a joint probability density function to describe these correlated failure modes. Thus, it is difficult to compute the reliability when considering the correlations between the failure modes. It is supposed that three or more failure modes arise synchronously to be a very small probability event. The relationship between ultimate state functions in different failure modes is established by utilizing linear regression method. A double integration model for reliability of mechanical components with dependent failure modes is built according to stress-strength interference model. In case of square, cube, or exponential relationship between two ultimate state functions, a linear transformation is made. An example of pin that may fail in shear fracture, bruise, or both is discussed. The reliability is compared with that obtained by using Monte Carlo method, which represents that the reliability model with dependent failure modes proposed is correct.
\end{abstract}

\section{Introduction}

Mechanical components may fail in many modes such as fatigue, creep, wear, resonance, plastic deformation, and instability which lead to several ultimate state functions. The ultimate state functions are often functions of the same random variables; therefore, they are not independent, and there may be some complex or even unknown relations between them. There is generally not a joint probability density function to describe these correlated failure modes; thus, it is a difficult and hot issue to compute the reliability with dependent failure modes in reliability study [1-3]. In [4-7], each failure mode is assumed to be independent when calculating the reliability with many failure modes; then, the series model of system reliability theory is introduced. This method is thought to be less rigorous in theory because the relevance of ultimate state functions among different failure modes is ignored, and the calculation error caused by it is hard to estimate. According to the relevance between the two failure modes, the bound method of reliability calculation is proposed in [8], which distinguishes the two failure modes into primary failure mode and secondary failure mode; the two failure modes are assumed to be of complete correlation and complete dissociation, and the actual condition of the structure falls in between the two modes, and thus the interval estimation value of relevant reliability is obtained. This method is easy to compute and widely used in the reliability design of civilian industry product $[9,10]$; however, it belongs to qualitative analytical method, and only the interval estimation value can be acquired. In [11, 12], the correlation coefficient is applied to characterize the relationship among different failure modes as the nuclear power stations do for the reliability design of critical parts in many failure modes; if they are positively correlated and the correlation is desirable, a satisfying result can be obtained; if they are of negative correlation or the correlation is poor, larger error is obtained. Actually, the correlation coefficient can just illustrate the two failure modes to the extent of linear and cannot represent the real relation between them [13, 14]; for example, $X^{2}+Y^{2}=1$, where $X$ and $Y$ are strongly correlated, but the correlation coefficient between them is 0 . Errors will be introduced when using correlation coefficient to deal with the problems of reliability calculation in many failure modes. In $[15,16]$, the Copula function is used to describe the correlation of the 
random variables, and the Copula-Reliability method with dependent failure modes is obtained in which the multiple integral operation is replaced by multiple differential operation to simplify the reliability calculation process; but the approximate correlated relation between each failure modes must be identified firstly to reasonably choose Copula model during the implementation process of this method. Moreover, more sample values and some parameter estimation skills are needed to confirm the relevant parameter. The Monte Carlo method is used to calculate the reliability model of components and systems with dependent failure modes in $[17,18]$. It needs no assumption or simplicity of the relation among failure modes, basic random variables, and ultimate state functions, and thus it has a wide application scope; however, when the reliability is high or the number of random variables is large, it needs higher sampling amount. Therefore, the Monte Carlo method is usually used to verify the theory method [14].

It is supposed in this paper that three or more failure modes existing simultaneously is a minimum probability event, the functional relation between ultimate state functions in different failure modes is obtained through linear regression method, double integration model for reliability is established by imitating the stress-strength interference model, and the Monte Carlo method is used to verify the new reliability model.

\section{Reliability Model with Multiple Failure Modes}

Suppose that a mechanical component is controlled by the vector of random variables $\mathbf{X}$,

$$
\mathbf{X}=\left[X_{1}, X_{2}, \ldots, X_{H}\right] .
$$

$X_{h}(h=1,2, \ldots, H)$ are the basic random variables. Assume that there are $m$ probable failure modes of the mechanical components, and the relative ultimate state functions are

$$
g_{1}(\mathbf{X})=0, g_{2}(\mathbf{X})=0, \ldots, g_{m}(\mathbf{X})=0,
$$

where $g_{i}(\mathbf{X})(i=1,2, \ldots, m)$ are supposed to obey normal distribution for simplicity. Take the event that the $i$ th failure mode occurs $\left(g_{i}(\mathbf{X})<0\right)$ as $E_{i}$, and the failure probability $F$ of the components can be presented as

$$
F=\sum_{i=1}^{m} P\left(E_{i}\right)-\sum_{1 \leq i<j \leq m} P\left(E_{i} E_{j}\right)+\cdots+(-1)^{m-1} P\left(E_{1} E_{2} \cdots E_{m}\right) .
$$

Considering that three or more failure modes occur synchronously is an event of very small probability, (3) can be expressed as

$$
F \approx \sum_{i=1}^{m} P\left(E_{i}\right)-\sum_{1 \leq i<j \leq m} P\left(E_{i} E_{j}\right) .
$$

Failure probability for single failure mode of the components can be obtained as follows:

$$
P\left(E_{i}\right)=\int_{-\infty}^{0} f_{i}\left(g_{i}\right) \mathrm{d} g_{i}
$$

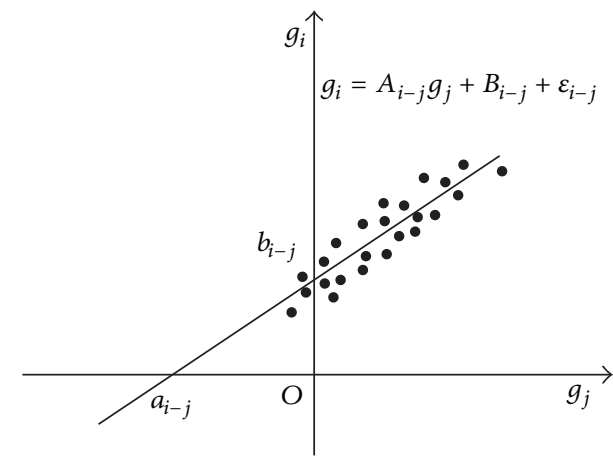

FIGURE 1: Relationship of ultimate state functions of different failure modes.

where $f_{i}\left(g_{i}\right)$ are the ultimate state functions, and $g_{i}$ are the probability density functions.

There are some inevitable functional relationships between generalized strength and generalized stress for the same components in each failure mode; therefore, the probability that $E_{i}$ and $E_{j}$ occur simultaneously cannot be calculated directly. Suppose that the relationships of ultimate state functions of each failure mode are approximately linear, as is shown in Figure 1. Even if there are other functional relationships between them, it can be translated into linear relationship through linear conversion.

The linear function can be presented as

$$
g_{i}=A_{i-j} g_{j}+B_{i-j}+\varepsilon_{i-j},
$$

where $A_{i-j}, B_{i-j}$ are constants; $\varepsilon_{i-j}$ obey normal distribution $N\left(0, \sigma_{i-j}\right)$, and the intercept of the line on $g_{j}$ axis is recorded as $a_{i-j}$.

In order to obtain the distribution function of $a_{i-j}$, linear regression analysis is introduced on the $N$ sets of observed value $\left(g_{j}^{n}, g_{i}^{n}\right)(n=1,2, \ldots, N)$ of $g_{j}, g_{i}$, and the estimation value of the unknown parameter in the straight line equation is acquired. Consider

$$
\begin{gathered}
\widehat{A}_{i-j}=\frac{\sum_{n=1}^{N} g_{j}^{n} g_{i}^{n}-(1 / N)\left(\sum_{n=1}^{N} g_{j}^{n} \sum_{n=1}^{N} g_{i}^{n}\right)}{\sum_{n=1}^{N}\left(g_{j}^{n}\right)^{2}-(1 / N)\left(\sum_{n=1}^{N} g_{j}^{n}\right)^{2}}, \\
\widehat{B}_{i-j}=\frac{\sum_{n=1}^{N} g_{j}^{n} \sum_{n=1}^{N}\left(g_{i}^{n}\right)^{2}-\sum_{n=1}^{N} g_{j}^{n} g_{i}^{n} \sum_{n=1}^{N} g_{j}^{n}}{N\left[\sum_{n=1}^{N}\left(g_{j}^{n}\right)^{2}-(1 / N)\left(\sum_{n=1}^{N} g_{j}^{n}\right)^{2}\right]} \\
\widehat{\sigma}_{i-j}^{2}=\frac{1}{N} \sum_{n=1}^{N}\left(g_{i}^{n}-\frac{1}{N} \sum_{n=1}^{N} g_{i}^{n}\right)^{2}-\frac{\widehat{A}_{i-j}^{2}}{N} \sum_{n=1}^{N}\left(g_{j}^{n}-\frac{1}{N} \sum_{n=1}^{N} g_{j}^{n}\right)^{2} .
\end{gathered}
$$
is

Notice that the intercept $a_{i-j}$ of the fitting line on $g_{j}$ axis

$$
\widehat{a}_{i-j}=-\frac{\widehat{B}_{i-j}+\varepsilon_{i-j}}{\widehat{A}_{i-j}} .
$$

It is thus clear that $\widehat{a}_{i-j}$ obey normal distribution $N\left(-\widehat{B}_{i-j} / \widehat{A}_{i-j}, \widehat{\sigma}_{i-j} / \widehat{A}_{i-j}\right)$. 


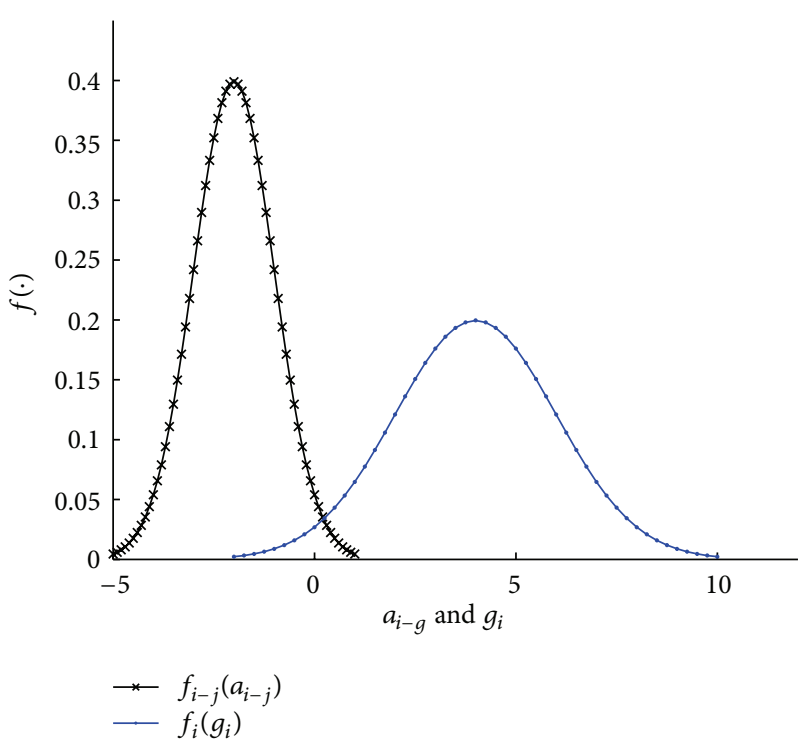

FIGURE 2: Relationship of $g_{j}$ and $a_{i-j}$ distribution functions.

It can be concluded from Figure 1 that

(1) if $A_{i-j}>0$;

$$
\begin{aligned}
& P\left(E_{i} E_{j}\right) \\
& \quad=P\left[\left(g_{j}<a_{i-j} \leq 0\right) \cup\left(g_{j}<0 \cap a_{i-j}>0\right)\right] \\
& \quad=P\left(g_{j}<a_{i-j} \leq 0\right)+P\left(g_{j}<0 \cap a_{i-j}>0\right) ;
\end{aligned}
$$

(2) if $A_{i-j}<0$;

$$
P\left(E_{i} E_{j}\right)=P\left(a_{i-j}<g_{j} \leq 0\right) .
$$

The distribution curves of $g_{j}, a_{i-j}$ are presented in Figure 2 , in which $f_{i-j}\left(a_{i-j}\right)$ and $f_{i}\left(g_{i}\right)$ denote the probability density function of $g_{j}$ and $a_{i-j}$, respectively.

It can be concluded from Figure 2 that

$$
\begin{aligned}
& P\left(g_{j}<a_{i-j}<0\right)=\int_{-\infty}^{0} f_{i-j}\left(a_{i-j}\right)\left[\int_{-\infty}^{a_{i-j}} f_{i}\left(g_{j}\right) \mathrm{d} g_{j}\right] \mathrm{d} a_{i-j}, \\
& P\left(g_{j}<0 \cap a_{i-j}>0\right)=\int_{-\infty}^{0} f_{i}\left(g_{j}\right) \mathrm{d} g_{j} \int_{0}^{+\infty} f_{i-j}\left(a_{i-j}\right) \mathrm{d} a_{i-j}, \\
& P\left(a_{i-j}<g_{j} \leq 0\right)=\int_{-\infty}^{0} f_{i}\left(g_{j}\right)\left[\int_{-\infty}^{g_{i}} f_{i-j}\left(a_{i-j}\right) \mathrm{d} a_{i-j}\right] \mathrm{d} g_{j} .
\end{aligned}
$$

Equations (11)-(13) can be solved through numerical integration method.

\section{Validation of Reliability Model with Dependent Failure Modes Utilizing Monte Carlo Method}

The Monte Carlo method is also called stochastic simulation [19]. It is a numerical computation method that is on the basis of probability statistics theory, in terms of the laws of large numbers, with the utilization of numerical simulation technology and through statistical sampling test or stochastic simulation of relevant random variable to seek for approximate solution of complex engineering and technology problems. The basic idea of the method is first to build the probability model of the problem to be solved, then produce a large number of sample values of random variable according to the statistical property of random variable of probability model, and last through sampling calculation of probability model to get the approximate solution of the problem to be solved. Mathematical difficulties in structure reliability analysis can be avoided in Monte Carlo simulation method, and no matter if the state functions are nonlinear or not, the random variables are normal distribution or not, and the limit state functions are correlated or not, as long as the number of simulation is large enough, accurate failure probability and reliability index can be obtained. Thus, the Monte Carlo simulation method is used to assess the correctness of the new method in this paper.

The hypothesis that three or more failure modes exist simultaneously is a minimum probability event which is unnecessary when using the Monte Carlo method to calculate the reliability with dependent failure modes, and sampling calculation of (14) can be directly used. Consider

$$
R=P\left(\bigcap_{i=1}^{m} \bar{E}_{i}\right)
$$

Concrete steps are listed as follows:

(1) initialize loop control variable $i i$, sampling amount $r$ and safe times $R g$, in which $r$ is a larger value and the others are zero;

(2) generate $N$ dimensions vector of random number $\mathbf{X}($ ii $)$ according to the basic random variables $X_{h}(h=$ $1,2, \ldots, N)$;

(3) calculate $g_{1}^{(i i)}, g_{2}^{(i i)}, \ldots, g_{m}^{(i i)}$;

(4) test if $\bigcap_{i=1}^{m} g_{i}^{(i i)}>0(i=1,2, \ldots, m)$ is satisfied, and if it is the case,

$$
R g^{(i i+1)}=R g^{(i i)}+1
$$

(5) test if $i i \geq r$ is satisfied, and if it is the case, the reliability is

$$
R=\frac{R g}{r}
$$

If it is not case, $i i=i i+1$, back to the second step;

(6) output reliability $R$. 


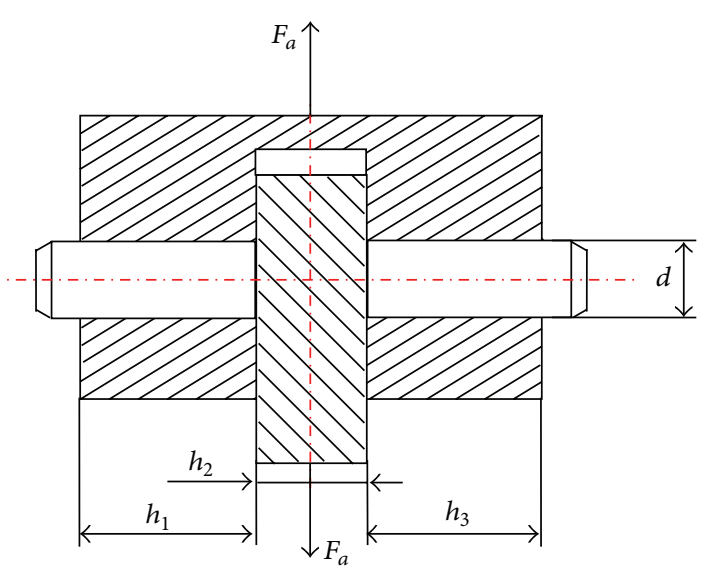

FIGURE 3: A pin connection component structure.

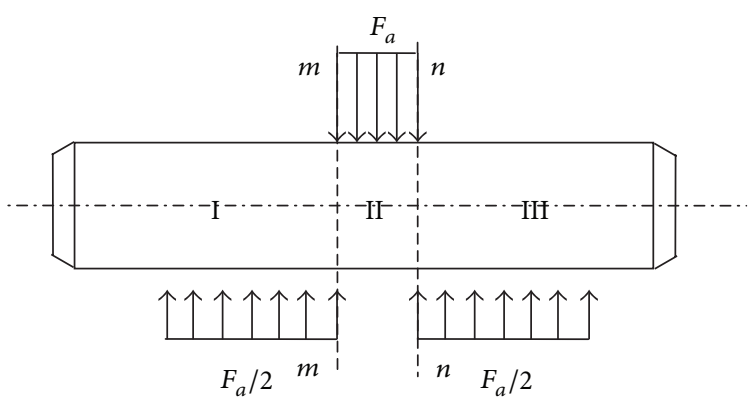

(a)
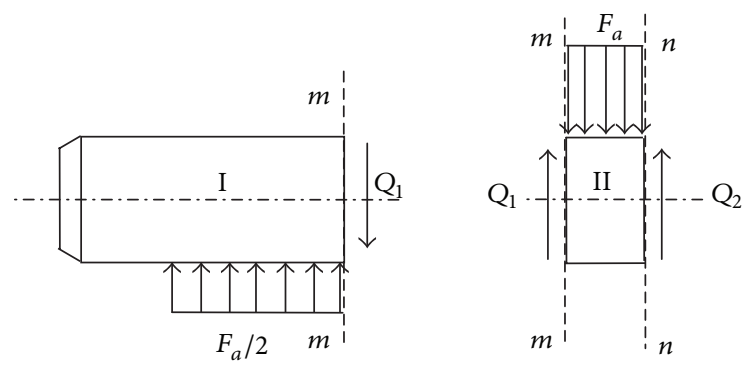

(b)

FIGURE 4: Force analysis of the pin.

The higher is the reliability, the more samples are required in the Monte Carlo method, and the Monte Carlo method is only used to verify the correctness of the model for reliability of components with dependent failure modes mentioned earlier.

\section{Examples}

Compute the reliability of the pin connection component in Figure 3. Given that $F_{a} \sim N\left(6000,1500^{2}\right) N$, the diameter of pin $d=10 \mathrm{~mm}, h_{1}=h_{3}=30 \mathrm{~mm}$, and $h_{2}=8 \mathrm{~mm}$. The shear strength $[\tau]$ and bearing strength of 20 pins are obtained by experiment and listed in Table 1.

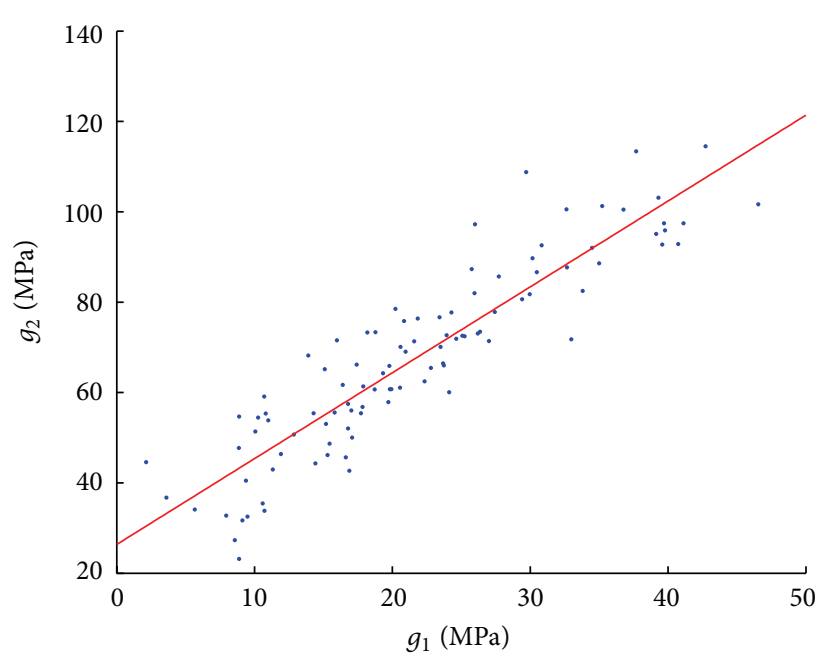

FIGURE 5: Functional relation between $g_{1}$ and $g_{2}$.

The solution of the problem is listed as follows.

(1) Force analysis. The force analysis of the pin is illustrated in Figure 4(a). The pin is divided into three parts I, II, and III with assumed plane $m-m$ and plane $n-n$. Because the force condition of part I is the same as part III, only part I and II are taken as research objects as shown in Figure 4(b). From equilibrium conditions, we can get $Q_{1}=Q_{2}=F_{a} / 2$. Obviously, the bearing force of part II is bigger and the bearing area is smaller. Therefore, only part II is possible to fail in the example.

(2) Failure mode analysis. The structure has two single failure modes. They are, respectively, $E_{1}$ : shear fracture of the pin and $E_{2}$ : bruise of the pin. Moreover, the structure is possible to have two failure modes synchronously. Therefore, the failure probability $F$ is

$$
F=P\left(E_{1}\right)+P\left(E_{2}\right)+P\left(E_{1} E_{2}\right) .
$$

(3) The limit state functions of the two failure modes are

$$
\begin{aligned}
& g_{1}=[\tau]-\frac{2 F_{a}}{\pi d^{2}}=[\tau]-0.0064 F_{a}, \\
& g_{2}=\left[\sigma_{j y}\right]-\frac{F_{a}}{d h_{2}}=\left[\sigma_{j y}\right]-0.0125 F_{a} .
\end{aligned}
$$

The shear strength of the pin $[\tau] \sim N\left(60.5,3^{2}\right)$ $\mathrm{MPa}$ and the bearing strength of the pin $\left[\sigma_{j y}\right]_{1} \sim$ $N\left(162.9,6.1^{2}\right) \mathrm{MPa}$ are both obtained by statistics data. The relationship between $[\tau]$ and $\left[\sigma_{j y}\right]_{1}$ is shown in Figure 4, and the relationships between the two limit state functions are

$g_{1} \sim N(21.93,9.90) ; \quad g_{2} \sim N(69.42,20.72)$. 
Table 1: The Statistics Data of Shear Strength and Bearing Strength of 20 Pins.

\begin{tabular}{lcccccccccc}
\hline Serial number & 1 & 2 & 3 & 4 & 5 & 6 & 7 & 8 & 9 \\
\hline$[\tau] / \mathrm{MPa}$ & 60.36 & 61.84 & 56.73 & 58.71 & 59.27 & 56.60 & 56.31 & 62.61 & 60.03 & 58.20 \\
{$\left[\sigma_{j y}\right] / \mathrm{MPa}$} & 142.40 & 143.30 & 138.45 & 146.37 & 139.34 & 132.82 & 134.63 & 150.87 & 159.65 & 154.58 \\
Serial number & 11 & 12 & 13 & 14 & 15 & 16 & 17 & 18 & 19 & 20 \\
{$[\tau] / \mathrm{MPa}$} & 62.26 & 60.65 & 57.23 & 63.75 & 60.81 & 64.14 & 63.19 & 58.08 & 56.38 & 59.80 \\
{$\left[\sigma_{j y}\right] / \mathrm{MPa}$} & 167.03 & 144.04 & 139.4 & 148.12 & 144.02 & 149.54 & 149.06 & 145.31 & 133.34 & 137.20 \\
\hline
\end{tabular}

(4) According to the distribution pattern of the basic variables, the accounted functional relation between $g_{1}$ and $g_{2}$ is shown in Figure 5.

The unknown parameters are obtained from (7) as

$$
\widehat{A}_{2-1}=1.90 ; \quad \widehat{B}_{2-1}=26.36 ; \quad \sigma_{2-1}^{2}=75.45 \text {, }
$$

and intercept $a_{i-j}$ is obtained from (8) as

$$
a_{1-2} \sim N(-13.87,4.57) \text {. }
$$

(5) Calculation of failure probability $F$ and reliability $R$. According to the standard normal distribution list, the failure probabilities $P\left(E_{1}\right)$ and $P\left(E_{2}\right)$ are

$$
\begin{aligned}
& P\left(E_{1}\right)=\Phi\left(\frac{0-21.93}{9.90}\right)=1.39 \times 10^{-2}, \\
& P\left(E_{2}\right)=\Phi\left(\frac{0-69.42}{20.72}\right)=5.38 \times 10^{-4} .
\end{aligned}
$$

The failure probability $P\left(E_{1} E_{2}\right)$ can be obtained from (9), (11), and (12) as

$$
\begin{gathered}
P\left(E_{1} E_{2}\right)=\int_{-\infty}^{0} \frac{1}{4.57 \sqrt{2 \pi}} \exp \left[-\frac{1}{2}\left(\frac{a_{2-1}+13.87}{4.57}\right)^{2}\right] \\
\left\{\int_{-\infty}^{a_{2-1}} \frac{1}{9.90 \sqrt{2 \pi}} \exp \left[-\frac{1}{2}\left(\frac{g_{1}-21.93}{9.90}\right)^{2}\right] \mathrm{d} g_{1}\right\} \mathrm{d} a_{2-1} \\
+\int_{-\infty}^{0} \frac{1}{9.90 \sqrt{2 \pi}} \exp \left[-\frac{1}{2}\left(\frac{g_{1}-21.93}{9.90}\right)^{2}\right] \mathrm{d} g_{1} \\
\int_{0}^{+\infty} \frac{1}{4.57 \sqrt{2 \pi}} \exp \left[-\frac{1}{2}\left(\frac{a_{2-1}+13.87}{4.57}\right)^{2}\right] \mathrm{d} a_{2-1} \\
=3.04 \times 10^{-4}+1.61 \times 10^{-5}=3.20 \times 10^{-4} \\
F=P\left(E_{1}\right)+P\left(E_{2}\right)-P\left(E_{1} E_{2}\right)=1.41 \times 10^{-2}, \\
R=1-F=0.9859 .
\end{gathered}
$$

(6) According to (4), conducting $10^{5}$ samples with the method of Monte Carlo, the reliability $R=0.985613$; when the sampling amount is $10^{6}$, the reliability $R=$ 0.98618 , and when the sampling amount is $1.5 \times 10^{7}$, the reliability $R=0.985943$.

It is clear that the reliability of the pin obtained from the theoretical approach and Monte Carlo simulation shows good agreements; therefore, the double integration model for reliability of mechanical components with dependent failure modes established in this paper is correct.

\section{Conclusion}

(1) It is supposed that three or more failure modes arise synchronously to be a minimum probability event. The relationship between different ultimate state functions with dependent failure modes is established with the method of linear regression; the double integration model for reliability of mechanical components with dependent failure modes is established by imitating the stress-strength interference model, and it is verified with the method of Monte Carlo.

(2) For the simplicity and ease of description of the problem, suppose that the relationship between ultimate state functions is linear. In case of square, cubic, or exponential relationship, linear transformation is used before utilizing this model.

\section{Acknowledgments}

This project is supported by the Ministry of Major Science and Technology of Liaoning (Grant no. 2012219020) and Shenyang Key Scientific and Technological Program (Grant no. F12-006-2-00).

\section{References}

[1] S. Zhili and Z. Naisu, "Reliability calculation of a machine component failed in different modes," Journal of Northeastern University, vol. 35, no. 3, pp. 5-8, 2004.

[2] Y. Zhang, S. Wang, Q. Liu, and B. Wen, "Reliability analysis of multi-degree-of-freedom nonlinear random structure vibration systems with correlation failure modes," Science in China E, vol. 46, no. 5, pp. 498-508, 2003.

[3] Y. Zhang, X. Zhang, and X. Huang, "Robust reliability-based design optimization of mechanical components with multiple failure modes," China Mechanical Engineering, vol. 20, no. 2, pp. 142-149, 2009.

[4] D. F. Frost and K. F. Poole, "A method for predicting VLSIdevice reliability using series models for failure mechanisms," IEEE Transactions on Reliability, vol. 36, no. 2, pp. 234-242, 1987.

[5] W. K. Chung, "Reliability analysis of a series repairable system with multiple failures," Microelectronics Reliability, vol. 31, no. 2-3, pp. 371-373, 1991.

[6] M. Kodama and I. Sawa, "Reliability and maintainability of a multicomponent series-parallel system with simultaneous 
failure and repair priorities," Microelectronics Reliability, vol. 24, no. 1, pp. 147-164, 1984.

[7] D. Panko, T. Vanhoucke, R. Campos, and G. A. M. Hurkx, "Time-to-fail extraction model for the "mixed-mode" reliability of high-performance SiGE bipolar transistors," in Proceedings of the 44th Annual IEEE International Reliability Physics Symposium (IRPS '06), pp. 512-515, March 2006.

[8] O. Ditlevsen, "Narrow reliability bounds for structural system," Journal of Structural Mechanics, vol. 7, no. 4, pp. 453-472, 1979.

[9] I. Kozine and V. Krymsky, "An interval-valued reliability model with bounded failure rates," International Journal of General Systems, vol. 41, no. 8, pp. 760-773, 2012.

[10] M. Boushaba and Z. Azouz, "Reliability bounds of a 3dimensional consecutive-k-out-of-n : F system," International Journal of Reliability, Quality and Safety Engineering, vol. 18, no. 1, pp. 51-59, 2011.

[11] Y. Zhou and Z. Yimin, "Reliability sensitivity-based correlation coefficient calculation in structural reliability analysis," Chinese Journal of Mechanical Engineering, vol. 25, no. 3, pp. 608-614, 2012.

[12] H. Xianzhen, "Probabilistic approach to system reliability of mechanism with correlated failure models," Mathematical Problems in Engineering, vol. 2012, Article ID 465853, 11 pages, 2012.

[13] F. Steiner and B. Hajagos, "Reliability of the estimations of the correlation coefficient," Acta Geodaetica et Geophysica Hungarica, vol. 36, no. 4, pp. 399-416, 2001.

[14] B. Radim and G. Martorell, Reliability, Risk and Safety, CRC Press, Boca Raton, Fla, USA, 2009.

[15] A. Singh and Z. P. Mourelatos, "Time-dependent reliability estimation for dynamic systems using a random process approach," SAE International Journal of Materials and Manufacturing, vol. 3, no. 1, pp. 339-355, 2010.

[16] Y. Sun, P. Wang, L. Cheng, and H. Liu, “Operational reliability assessment of power systems considering condition-dependent failure rate," IET Generation, Transmission and Distribution, vol. 4, no. 1, pp. 60-72, 2010.

[17] R. C. Kuczera, Z. P. Mourelatos, and M. Latcha, "A Monte Carlo reliability assessment for multiple failure region problems using approximate metamodels," in Proceedings of the ASME International Design Engineering Technical Conferences and Computers and Information in Engineering Conference (IDETC '07), pp. 1135-1147, September 2007.

[18] T. Solver and M. Amelin, "State duration based Monte Carlo Simulation model with independent failures for distribution system reliability analysis," in Proceedings of the 9th International Conference on Probabilistic Methods Applied to Power Systems (PMAPS '06), pp. 1-8, June 2006.

[19] P. David and K. Binder, A Guide to Monte Carlo Simulations in Statistical Physics, Cambridge University Press, New York, NY, USA, 2010. 


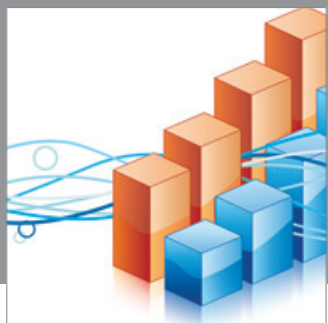

Advances in

Operations Research

mansans

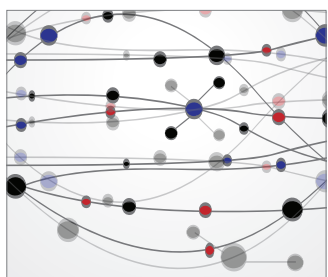

The Scientific World Journal
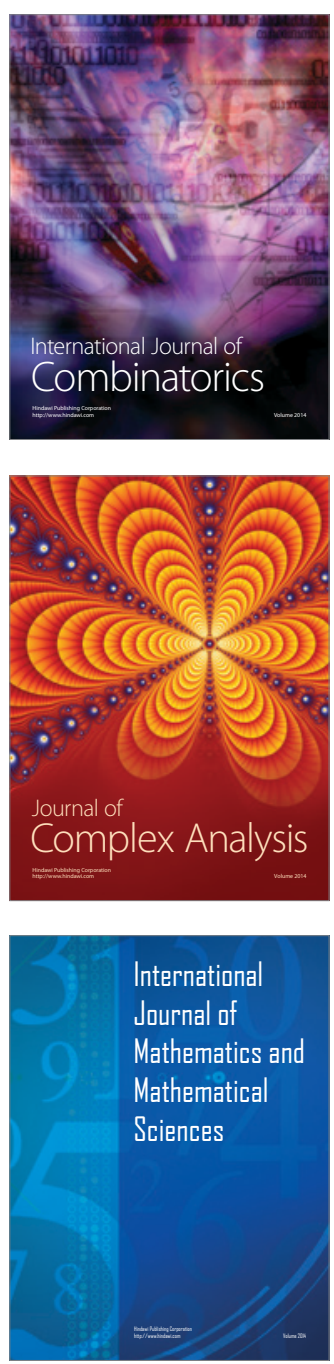
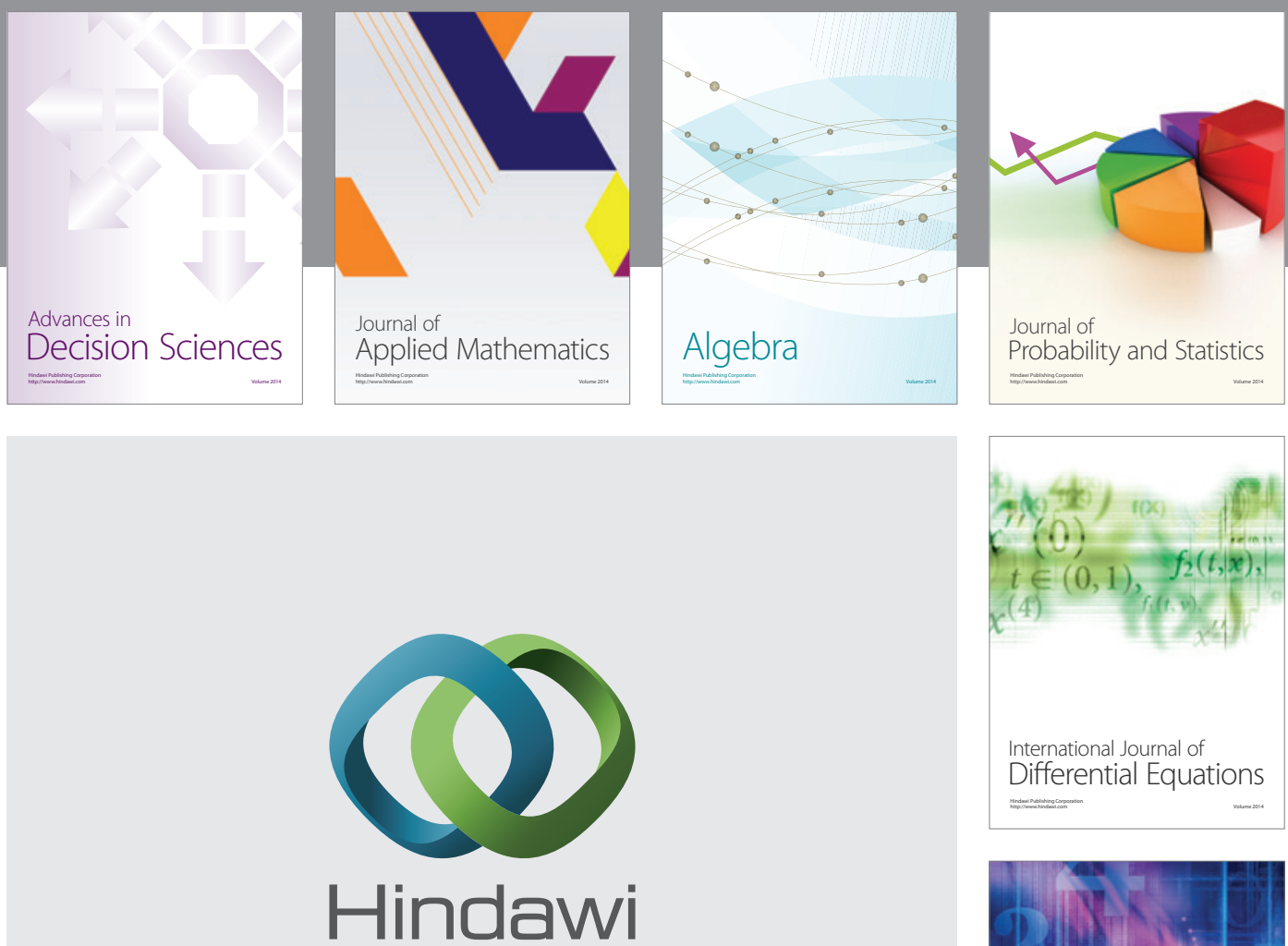

Submit your manuscripts at http://www.hindawi.com
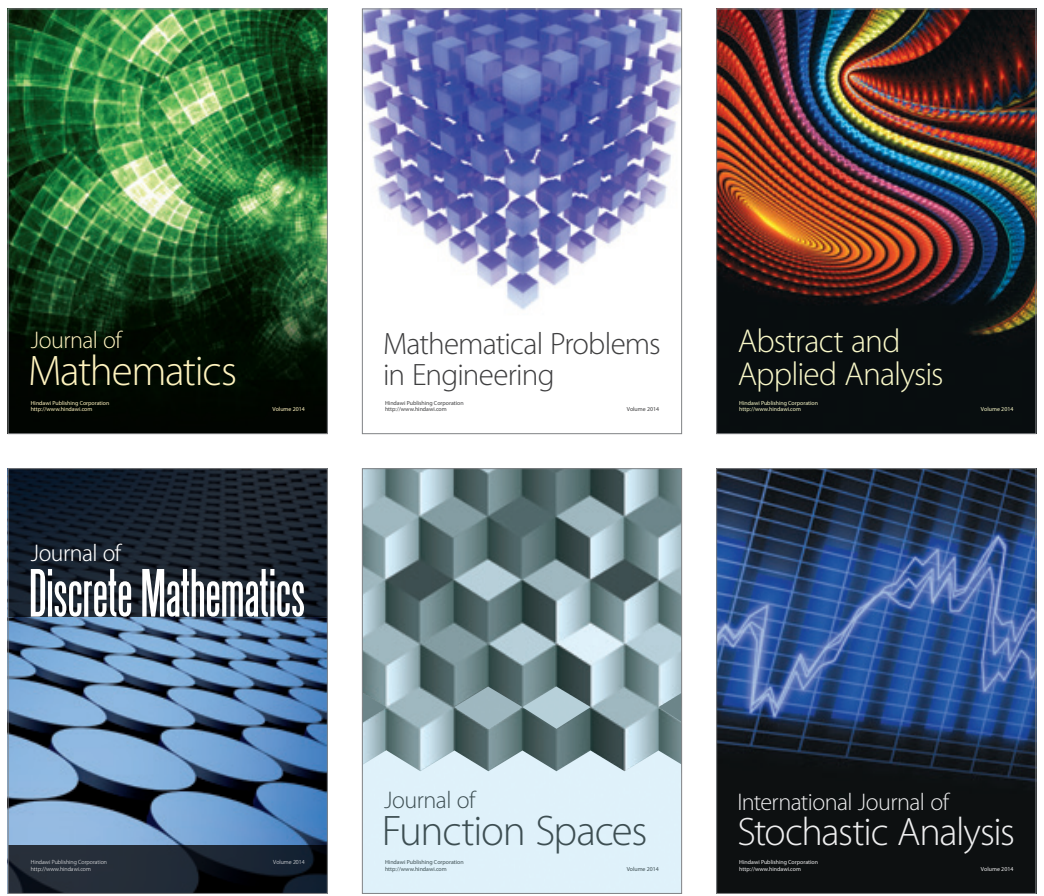

Journal of

Function Spaces

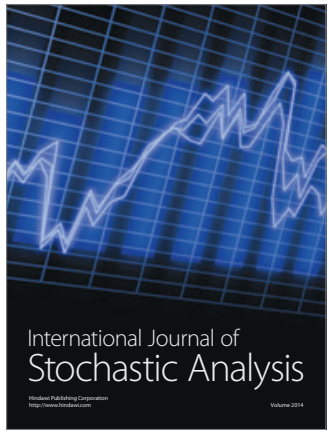

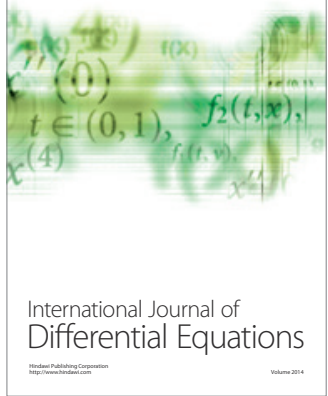
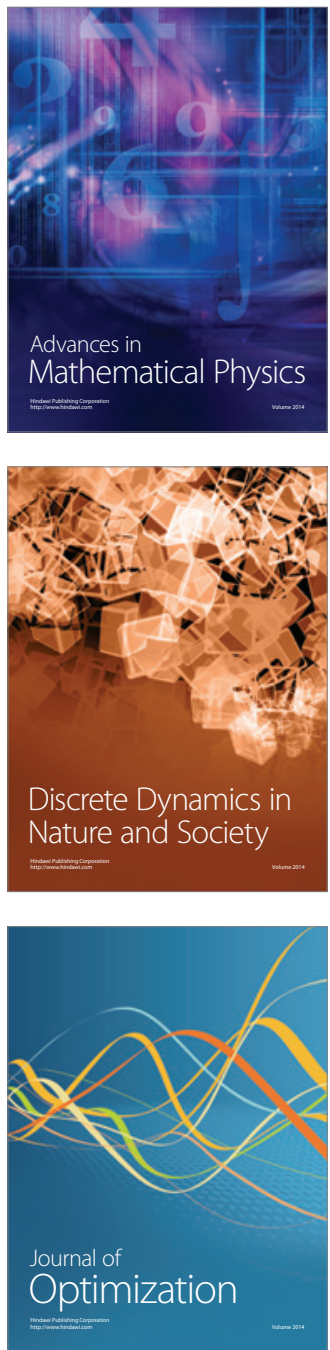